\title{
Apoptosis of Human Vascular Smooth Muscle Cells Derived from Normal Vessels and Coronary Atherosclerotic Plaques
}

\author{
Martin R. Bennett, * Gerard I. Evan, ${ }^{\ddagger}$ and Stephen M. Schwartz \\ *Department of Pathology, University of Washington, Seattle, Washington 98195; and ${ }^{\ddagger}$ Biochemistry of the Cell Nucleus Laboratory, \\ Imperial Cancer Research Fund, London, WC2A 3PX United Kingdom
}

\begin{abstract}
We studied death of human vascular smooth muscle cells derived from coronary plaques and normal coronary arteries and aorta. Cells from normal arteries underwent death only upon removal of serum growth factors. In contrast, plaque-derived cells died even in high serum conditions, and death increased after serum withdrawal. Death was characteristically by apoptosis in both normal and plaquederived cells, as determined by time-lapse videomicroscopy, electron microscopy, and DNA fragmentation patterns. IGF-1 and PDGF were identified as potent survival factors in serum, whereas EGF and basic fibroblast growth factor had little effect. Stable expression of $b c l-2$, a protooncogene that regulates apoptosis in other cell lines, protected smooth muscle cells from apoptosis, although there was no detectable difference in endogenous $b c l-2$ expression between cells from plaques or normal vessels. We conclude that apoptosis of human vascular smooth muscle cells is regulated by both specific gene products and local cytokines acting as survival factors. Apoptosis may therefore regulate cell mass in the normal arterial wall and the higher rates of apoptosis seen in plaque smooth muscle cells may ultimately contribute to plaque rupture and breakdown and thus to the clinical sequelae of atherosclerosis. (J. Clin. Invest. 1995. 95:22662274.) Key words: apoptosis - atherosclerosis • vascular smooth muscle $\bullet b c l-2$
\end{abstract}

\section{Introduction}

Cell death is a prominent component of the human atherosclerotic plaque with areas of "necrosis" being present in over $80 \%$ of primary lesions $(1,2)$. Ultimately, the morbidity and mortality associated with atherosclerosis results from rupture of the plaque into this "necrotic" area, with thrombosis leading to both ischemia and infarction (3). Cell death is also present in animal models of atherosclerosis. In fat-fed swine, for instance, one of the most well-characterized models of cell kinetics in atherosclerosis, cell death is present at all stages of lesion development (4-6). In the early lesions in this model, cell

Dr. Martin R. Bennett, Unit of Cardiovascular Medicine, University of Cambridge School of Clinical Medicine, Department of Medicine, Level 5, Addenbrooke's Hospital, Hills Road, Cambridge CB22QQ, UK. Phone/FAX: 01223 331504/5.

Received for publication 6 September 1994 and in revised form 13 December 1994.

J. Clin. Invest.

(c) The American Society for Clinical Investigation, Inc. 0021-9738/95/05/2266/09 \$2.00

Volume 95, May 1995, 2266-2274 death balances cell proliferation, and no overall accumulation of tissue ensues (4). In later lesions, lipid rich necrotic areas are more prominent, with death occurring in both macrophages and vascular smooth muscle cells (VSMCs). ${ }^{1}$ Cell death in the vasculature has previously been thought to be due to direct toxic insult, as a reaction to free radical generation or peroxidation from oxidised lipids, for instance $(7,8)$. However, recent evidence has indicated that cell death of endothelial cells (9), VSMCs (10), and macrophages (11) may all occur via the highly regulated process of programmed cell death, or apoptosis.

Apoptosis is a ubiquitous, evolutionarily conserved, physiological mechanism of cell death that regulates tissue mass and architecture in many tissues (12). The genetic program for cell death, which is contained in selected or even all cells, may be activated at a defined time during embryogenesis or in the maturation of adult cells, by a wide range of physiological and pharmacological stimuli. In VSMCs, for example, we have identified apoptosis in the presence of deregulated expression of the $c-m y c$ protooncogene $(10,13)$. Deregulated expression of c-myc has previously been demonstrated to occur in one study of cultured human plaque VSMCs (14). The present study examined VSMCs derived from human coronary atheromatous plaques and from normal vessels for the presence of apoptosis. Our data demonstrate that passaged plaque VSMCs have a markedly elevated rate of apoptosis in vitro. Apoptosis of human VSMCs can be partially reversed by expression of the classical antiapoptotic gene $b c l-2$ or by gene products normally present in the arterial wall, IGF-1 and PDGF.

\section{Methods}

Cell culture. Normal human VSMCs were derived from the media of the thoracic aorta of donors for cardiac transplantation (four cultures) and from the media of coronary arteries of recipients undergoing transplantation for nonischemic cardiomyopathy (four cultures). Patients were in the University of Washington Medical Center transplantation program from 1987 to 1994 . Vessels were dissected from the heart, a piece of vessel taken for routine histology, and cells prepared by enzyme digestion of pieces of media. Briefly, arteries were predigested with an enzyme mix containing $165 \mathrm{U} / \mathrm{ml}$ collagenase type $\mathrm{I}, 15 \mathrm{U} / \mathrm{ml}$ elastase type III, $0.375 \mathrm{ng} / \mathrm{ml}$ of soybean trypsin inhibitor in serum-free Waymouth's medium for $20 \mathrm{~min}$ at room temperature. The vessels were then cut open and pinned luminal side up on a dissecting surface. The residual endothelium was removed with a plastic cell scraper and the media and intima separated by peeling the intima from the media. Medial tissue was then minced and digested in the enzyme mix for $16 \mathrm{~h}$ at $37^{\circ} \mathrm{C}$ until complete dispersion was obtained, as assessed by light microscopy. The remaining vessel was then fixed in $4 \%$ formaldehyde and processed for routine histology. The samples of vessel taken before and

1. Abbreviation used in this paper: VSMC, vascular smooth muscle cell. 
after dissection were used to confirm that the media had been cultured and that microscopic evidence of atherosclerosis in the form of foam cells, extracellular lipid, or matrix accumulation was not present.

Plaque VSMCs were obtained from patients who underwent directional coronary atherectomy (Simpson Coronary Atherocath, Devices for Vascular Intervention Inc., Redwood City, CA) in the cardiac catheterisation laboratory of the University of Washington Medical Center between 1990 and 1992. Specimens for culture were obtained from primary lesions (five cultures) and not from lesions that had undergone previous angioplasty or atherectomy. Multiple pieces of atherectomy tissue were obtained from each patient weighing between 10 and $30 \mathrm{~g}$ in total. The specimen was divided and one half fixed and processed for routine histology and orcein staining for elastin. The preparation of cells from atherectomy specimens followed the same protocol as that for normal medial cells except that the atherectomy tissue was minced and enzyme digested for $16 \mathrm{~h}$. Cells from both atherectomy specimens and normal vessels were then plated in Waymouth's medium containing 20\% FCS, $10 \mathrm{mM}$ Hepes (Sigma Chemical Co., St. Louis, MO), 2\% ECGS (Sigma), and equilibrated with $95 \%$ air and $5 \% \mathrm{CO}_{2}$. Cultures from individual patients were maintained as separate cultures and cells were not pooled. Subconfluent cells were passaged by trypsinization in $0.05 \%$ trypsin in PBS. At passage three, cultures were analyzed immunocytochemically to confirm smooth muscle origin ( $\alpha$-actin positive, vimentin positive, vWf negative, desmin negative, and smooth muscle myosin negative).

Infection of cells with bcl-2. The retrovirus vector pbcl-2-neo was obtained from Dr. David Hockenbery (Fred Hutchison Cancer Center, Seattle, WA) and contained a cDNA encoding full-length human $b c l$ 2 (15). Infection of cells with retrovirus containing $b c l-2$ or the vector alone was performed as previously described (10). Passage four through six cells were plated in 10-cm-diameter dishes at $2 \times 10^{7}$ cells $/$ dish in normal tissue culture medium and infected using the retrovirus constructs. Cell plates were split 1:50 and cells were selected in medium containing $750 \mu \mathrm{g} / \mathrm{ml}$ of the neomycin analogue G418 (GIBCO, Grand Island, NY). After 1 wk of antibiotic treatment, isolated surviving single cells were evident on culture plates. Clones of resistant cells containing bcl-2 were ring cloned after 4-6 wk and both clones and an uncloned population were cultured in medium containing G418 thereafter. Clones of $b c l-2$-infected cells and cell populations were each tested individually and compared against a pooled population of cells infected with the vector alone. Cells were maintained in neomycin at all times and used for experiments after a minimum of $4 \mathrm{wk}$ in selection.

Analysis of cell number and flow cytometry. Cells were plated into 24-well plates at $1 \times 10^{4}$ cells/well in medium containing $20 \%$ FCS and growth of cultures were analyzed by cell counts over $10 \mathrm{~d}$ of cells that could exclude trypan blue. Cell cycle distribution of cells in culture in $20 \%$ FCS was determined by flow cytometric analysis as previously described (10).

Time-lapse videomicroscopy. Cells were prepared for videomicroscopy as previously described (10). Briefly, cells were maintained in medium containing $20 \%$ FCS, washed three times in medium containing $0 \% \mathrm{FCS}$, and then cultured in this latter medium. This was supplemented with selenite (30 nM), transferrin $(5 \mu \mathrm{g} / \mathrm{ml})$, fibronectin $(1 \mu \mathrm{g} / \mathrm{ml})$, and albumin $(1 \mathrm{mg} / \mathrm{ml})$. Flasks were gassed with $95 \%$ air and $5 \%$ $\mathrm{CO}_{2}$ every $24 \mathrm{~h}$ and sealed. The microscope was enclosed in a plastic environment chamber and maintained at $37^{\circ} \mathrm{C}$ by an external heater. The time-lapse equipment consisted of a camera (Dage-MTI Inc., Michigan, IN) and Panasonic 8050 time-lapse video recorder with Colorado video synchronization system (Colorado Video, Boulder, $\mathrm{CO}$ ). Films were analyzed for morphology of apoptosis and cell death rates as previously described $(10,13,16)$ using an observer blind to cell type and treatment conditions. Cell division was scored at the time at which septa appeared between two daughter cells and intermitotic time measured between divisions of successive generations derived from the same cell over an extended period (7-10 d). Apoptotic cell death events were scored midway between the last appearance of normality and the point at which the cell became fully detached and fragmented, an interval of typically 60-90 min. Each individual cell culture was analyzed in duplicate as a minimum (plaque VSMCs, $n=11$; normal coronary and aortic VSMCs, $n=8$ ).

Electron microscopy. VSMCs were cultured in medium containing $20 \%$ FCS for $48 \mathrm{~h}$ and then transferred to $0 \%$ FCS for $24 \mathrm{~h}$. Cultures were fixed in $3 \%$ glutaraldehyde in cacodylate buffer $(0.1 \mathrm{M}$ sodium cacodylate, $5 \%$ sucrose, $2 \mathrm{mM} \mathrm{CaCl}{ }_{2}$ for $30 \mathrm{~min}$ at room temperature, postfixed in $1 \%$ osmium tetroxide, dehydrated in a graded series of ethanol, and embedded in Medcast plastic. Sections were cut and stained in 6\% uranyl acetate and Reynold's lead stain. Cells were viewed on an electron microscope (model JEM 1200 Ex II, Jeol Ltd., Tokyo, Japan) at $80 \mathrm{~K}$ accelerating voltage.

Analysis for DNA fragmentation. For assessment of nucleosome laddering, $\sim 8 \times 10^{7}$ cells were cultured in medium containing $20 \%$ FCS until $50 \%$ confluent. A similar number of cells were cultured as above but then transferred to medium containing 0\% FCS. After $24 \mathrm{~h}$, cells appearing in the supernatant were centrifuged at $1,000 \mathrm{~g}$ for 5 min. Cell lysis, DNA extraction, electrophoresis, and staining were as previously described $(10)$.

Requirement for individual growth factors. To assess the effects of individual growth factors in the regulation of apoptosis, cells were prepared for time-lapse videomicroscopy as above, but individual growth factors were added back at concentrations of $0.1-100 \mathrm{ng} / \mathrm{ml}$ (PDGFAA or BB, IGF-1, EGF) or $1-1,000 \mathrm{ng} / \mathrm{ml}$ (basic fibroblast growth factor [bFGF]). All growth factors were recombinant human forms (Sigma). Cell deaths rates were assessed in triplicate for each concentration of each growth factor.

$R N A$ isolation and Northern hybridization. RNA was extracted from individual cultures of normal and plaque VSMCs cultured in 20\% FCS and from RL-7 cells, a human B-cell lymphoma cell line that has a $t(14 ; 18)$ chromosomal translocation and that overexpresses $b c l-2$. Total RNA from $10^{7}$ cells was isolated using the method of Chirgwin et al. (17). RNA was fractionated, transferred, and hybridized as previously described (18) using a random primed human bcl-2 probe, SFFV-bcl2 (15), and a random primed $28 \mathrm{~S}$ probe to verify equal loading of RNA.

Immunocytochemical analysis for Bcl-2 protein. VSMCs were plated onto eight-well Tissue-Tek slides (Nunc, Naperville, IL) at 3,000 cells/well and cultured for $48 \mathrm{~h}$. Cells were fixed and processed for immunocytochemistry as previously described (10). Briefly, cells were washed three times in PBS, fixed in 4\% paraformaldehyde in PBS at room temperature for $15 \mathrm{~min}$, and blocked using incubation buffer (PBS, $1 \%$ BSA, $0.5 \%$ Triton $X-100,0.02 \%$ sodium azide) for $30 \mathrm{~min}$ at room temperature. Bcl-2 protein was detected using a hamster monoclonal antibody $6 \mathrm{C} 8$ antibody (15) diluted to $1 \mu \mathrm{g} / \mathrm{ml}$ in incubation buffer and bound antibody visualized using a biotin-labeled horse anti-hamster antibody at 1:200 dilution and an avidin-biotin peroxidase detection system (Vector Labs, Burlingame, CA).

Statistical analyses. The means of apoptotic deaths were analyzed using ANOVA for multiple comparisons. Paired analysis between two groups, between plaque and normal VSMCs, or between uninfected and bcl-2-infected cell lines, for instance, was performed using Student's $t$-test where ANOVA indicated significance for the multiple comparison.

\section{Results}

Human VSMC cultures were obtained from coronary plaques (plaque VSMCs) and from the media of normal coronary arteries and aorta ("normal" VSMCs). Cultures were obtained from specimens from individual patients of both sexes and from a range of ages (Table I). Mean age of patients was $49.8 \mathrm{yr}$ (plaque), $41.8 \mathrm{yr}$ (normal aorta), and $47.8 \mathrm{yr}$ (normal coronary artery); these differences were not significant. Atherectomy specimens were from primary lesions only, which had not received previous intervention. Three out of five atherectomy specimens were from patients who were experiencing unstable angina, although by passages four through six no significant 
Table I. Details of Patients from Whom VSMC Cultures Were Obtained

\begin{tabular}{llllll}
\hline & Source & Age & Sex & Passage & \multicolumn{1}{c}{ Clinical status } \\
\hline & & $y r$ & & & \\
Plaque derived & & & & & \\
HASMC 66 & LAD & 49 & M & 6 & Stable angina \\
HASMC 103 & RCA & 50 & F & 4 & Unstable angina \\
HASMC 104 & RCA & 48 & M & 4 & Unstable angina \\
HASMC 105 & LAD & 57 & F & 4 & Stable angina \\
HASMC 120 & LCx & 45 & M & 4 & Unstable angina \\
Normal aorta & & & & & \\
HAMED 19 & Aorta & 53 & M & 6 & Motor vehicle accident \\
HAMED 39 & Aorta & 48 & M & 5 & Motor vehicle accident \\
HAMED 63 & Aorta & 16 & M & 4 & Motor vehicle accident \\
HAMED 88 & Aorta & 50 & F & 4 & Subarachnoid hemorrthage \\
Normal coronary & & & & & \\
artery & & & & & \\
HCMED 8753 & RCA & 34 & M & 4 & Nonischemic cardiomyopathy \\
HCMED 8758 & RCA & 47 & M & 4 & Nonischemic cardiomyopathy \\
HCMED 90103 & LCx & 48 & F & 4 & Nonischemic cardiomyopathy \\
HCMED 941 & LAD & 62 & M & 4 & Nonischemic cardiomyopathy \\
& & & & &
\end{tabular}

LAD, left anterior descending artery; LCx, left circumflex artery; RCA, right coronary artery; HASMC, human atherosclerotic smooth muscle cell; HAMED, human aortic medial smooth muscle cell; HCMED, Human coronary medial smooth muscle cell.

difference in proliferation or death rates could be found in plaque-derived cells from patients with stable or unstable angina (not shown). As in other studies using atherectomy specimens, the tissue retrieved was heterogeneous, consisting predominantly of loose connective tissue, with some areas of necrotic debris and calcification. In addition, small areas of the tissue consisted of amorphous acellular material consistent with old thrombus. In view of the nature of the atherectomy procedure and the nature of the tissue retrieved, it is likely that many anatomic regions of the plaque were sampled. However, in specimens processed for cell culture, the tissue did not contain structures identifiable as an internal elastic lamina or elastic lamellae, and the cellular component did not show the linear circumferential pattern of smooth muscle cells seen in the media. Thus, it is likely that only atherosclerotic intima of the artery was sampled in specimens that we have used for culture. Although it is known that the cells present in atherectomy specimens consist of smooth muscle cells, macrophages, $\mathrm{T}$ lymphocytes, and endothelial cells, after three passages in culture, only cells corresponding to a smooth muscle cell phenotype and immunocytochemical staining pattern were present (not shown).

The rate of cell death in each culture in high serum $(20 \%$ FCS), low serum (0\% FCS), and $0 \%$ FCS with the addition of specific growth factors was assessed by time-lapse videomicroscopy. Videomicroscopy is a sensitive method of measuring cell death, which, unlike cell counts or S-phase markers, can assess cell death separate from cell proliferation. In addition, as apoptosis exhibits a characteristic sequence of morphological changes on time-lapse videomicroscopy, this technique allows an assessment of mode of cell death. Apoptosis was further confirmed by electron microscopy and DNA laddering patterns. Death rates were compared in subconfluent cultures from native normal coronary, aortic and plaque VSMCs, and in cells stably infected with the $b c l-2$ protooncogene.

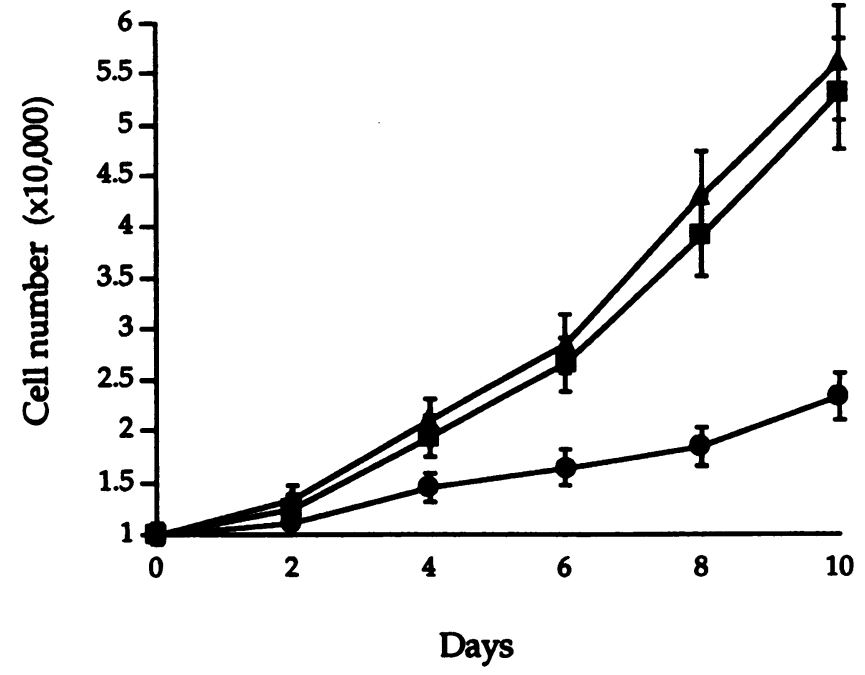

Figure 1. Increase in cell number over time in culture for plaque-derived and normal coronary and aortic VSMCs. VSMCs were plated at $1 \times 10^{4}$ cells/well in a 24-well plate in medium containing $20 \%$ FCS. Live cell number was assessed at the times shown by trypan blue exclusion. Values shown are means, error bars represent SEMs. Plaque-derived cells showed significantly slower increase in cell number than both normal VSMC types after $4 \mathrm{~d}(P<0.05)$. $\square$, normal aortic VSMCs; $\triangle$, normal coronary VSMCs; $\bullet$, plaque-derived VSMCs.

The proliferation of human VSMCs in culture. Before analysis of cell death of the human VSMC cultures, baseline kinetic parameters of each culture were determined at passage five and six. The rate of increase of cell number over time was analyzed in each cell type by cell counts, and the cell cycle distribution by flow cytometric analysis. This indicated that plaque-derived cells showed a significantly slower increase in cell number than cells derived from nonatherosclerotic aorta or coronary artery (Fig. 1). Consistent with this observation, the percentage of cells in S-phase in the plaque cell cultures in medium containing $20 \%$ FCS was significantly less than that of the normal cells (Table II). The intermitotic time of individual cells, assessed by extended time-lapse videomicroscopy, indicated that plaque-

Table II. Flow Cytometric Analysis of Cell Cycle Distribution and Intermitotic Time of Smooth Muscle Cell Cultures

\begin{tabular}{lcccc}
\hline \multicolumn{1}{c}{ Cell type } & GO/G1 & \multicolumn{1}{c}{$\mathrm{S}$} & $\mathrm{G} 2 \pm \mathrm{M}$ & $\begin{array}{c}\text { Intermitotic } \\
\text { time }\end{array}$ \\
\hline & & & & $h$ \\
Plaque cells & $88.5 \pm 6.8$ & $2.6 \pm 0.25$ & $8.9 \pm 1.2$ & $184.6 \pm 23.2$ \\
Normal aortic cells & $81.7 \pm 5.4$ & $7.9 \pm 1.7^{*}$ & $10.4 \pm 2.1$ & $98.2 \pm 16.4^{*}$ \\
$\begin{array}{l}\text { Normal coronary cells } \\
\text { Plaque bcl-2 cells }\end{array}$ & $82.1 \pm 7.9$ & $7.1 \pm 2.5^{*}$ & $10.8 \pm 1.7$ & $100.4 \pm 20.2^{*}$ \\
$\begin{array}{l}\text { Normal aorta bcl-2 } \\
\text { cells }\end{array}$ & $90.2 \pm 6.4$ & $2.2 \pm 0.9$ & $7.6 \pm 1.3$ & $202.2 \pm 21.7$ \\
$\begin{array}{l}\text { Normal coronary bcl-2 } \\
\text { cells }\end{array}$ & $84.2 \pm 5.8$ & $6.9 \pm 1.6^{*}$ & $8.9 \pm 1.1$ & $104.6 \pm 13.6^{*}$ \\
& $82.6 \pm 5.7$ & $8.2 \pm 2.8^{*}$ & $9.2 \pm 1.5$ & $106.5 \pm 18.4^{*}$ \\
& & & & \\
\end{tabular}

Values are means \pm SEM. Cells were cultured in medium containing $20 \%$ FCS and cells isolated after $\geq \mathbf{4 8} \mathrm{h}$ in culture. The mean intermitotic time of 20 cells from each culture type was assessed by extended time-lapse videomicroscopy, as the time between divisions of successive generations of cells. ${ }^{*} P<0.05$ vs plaque-derived cells. 


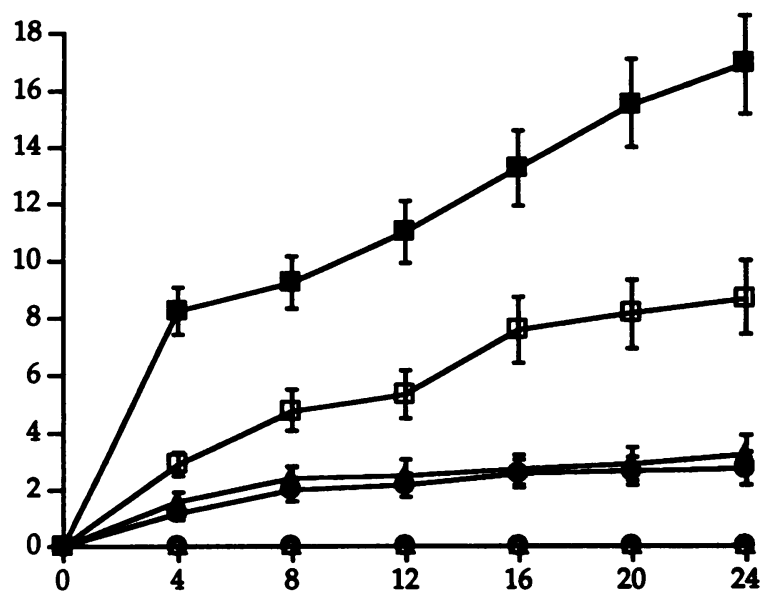

Time in hours

Figure 2. Cumulative number of cell deaths per 100 cells in plaque and normal VSMCs. Plaque-derived or normal coronary or aortic VSMCs were cultured in medium containing $20 \%$ or $0 \%$ FCS and deaths quantified by time-lapse videomicroscopy. Values given are means, error bars represent SEMs. Plaque cell deaths were significantly greater at time points after $4 \mathrm{~h}(P<0.05 \mathrm{vs}$. normal cells $) . \triangle$, normal coronary VSMCs in $20 \%$ FCS; $\triangle$, normal coronary VSMCs in 0\% FCS; $O$, normal aortic VSMCs in $20 \%$ FCS; $\bullet$, normal aortic VSMCs in 0\% FCS; $\square$, plaque VSMCs in $20 \%$ FCS; $\square$, plaque VSMCs in $0 \%$ FCS).

derived cells had a longer mean intermitotic time than both normal cell types (Table II). There was no significant difference in the growth parameters of human VSMCs derived from the media of coronary arteries or from the aorta.

Cell death of normal and plaque-derived VSMCs. Videomicroscopy of plaque and normal coronary and aortic VSMCs indicated a marked difference in the frequency of cell death between the cell types. Cells from normal coronary arteries or aorta did not undergo spontaneous cell death when cultured in $20 \%$ FCS. However, $2.7 \pm 0.5 \%$ (mean \pm SEM) of aortic cells and $3.25 \pm 0.4 \%$ of normal coronary cells died over $24 \mathrm{~h}$ in 0\% FCS (Fig. 2). In contrast, plaque-derived cells showed a significantly higher rate of spontaneous cell death in $20 \%$ FCS over $24 \mathrm{~h}(8.7 \pm 1.8 \%)$ and this increased to $16.8 \pm 2.7 \%$ in low serum. Cells were analyzed for rates of apoptosis in subconfluent culture, between passages four and six. However, there was no significant difference in the rate of cell death between these passages of the same culture (not shown). Death in all cell types exhibited rapid, sequential, morphological changes indicative of apoptosis $(10,16,19)$ (Fig. 3). Cells first retracted from their neighbors and shrunk in size. This was followed by intense membrane blebbing and the formation of membranebound vesicles. The end product was a dense apoptotic body, which in some cases was seen to be phagocytosed by adjacent cells. These appearances were confirmed on electron microscopy (Fig. 4, $A-D$ ), which in addition demonstrated peripheral chromatin condensation in association with the intense membrane activity. When DNA was extracted from cells in the culture supernatant, a nucleosome ladder pattern, indicative of internucleosomal cleavage, was evident in plaque cells in low and high serum and in normal VSMCs in low serum conditions

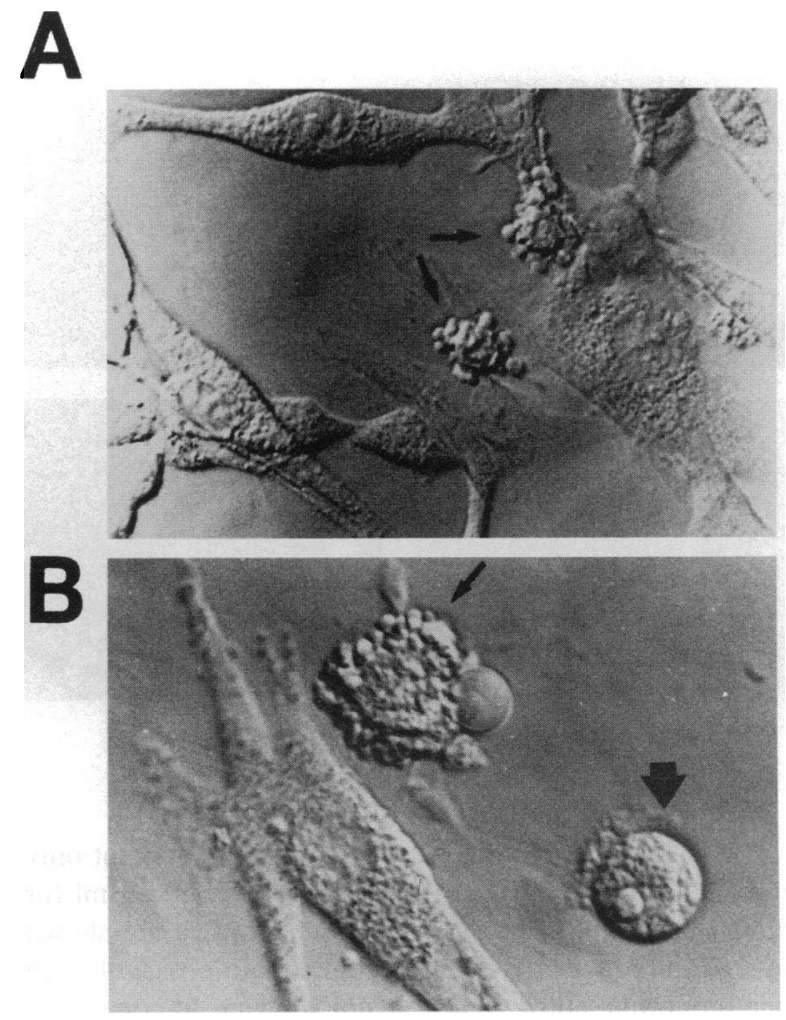

Figure 3. Time-lapse videomicroscopic appearances of human plaquederived VSMCs undergoing apoptosis in culture. $(A)$ Low power and $(B)$ high power. Cells first show retraction and shrinkage followed by formation of intense membrane blebs and membrane-bound vesicles (small arrows), with eventual formation of a dense apoptotic body (large arrow), which may be phagocytosed by adjacent cells.

(Fig. 5). This is consistent with apoptosis observed by timelapse videomicroscopy in plaque VSMCs in low and high serum but only in normal VSMCs in low serum (Fig. 2).

The effect of growth factor addition on apoptotic rates. Apoptosis of VSMCs in low serum might be either due to privation of essential nutrients or cofactors or to the absence of specific survival cytokines. To discriminate between these two possibilities, cells were first cultured in 0\% FCS but supplemented either with essential serum nutrients or with specific serum cytokines. Neither the addition of selenite, albumin, fibronectin, and transferrin nor the cytokine-free serum substitute CSPR-2 (Sigma) suppressed apoptosis in serum-deprived plaque or normal coronary or aortic VSMCs (not shown). To confirm that apoptosis was due to the removal of survival cytokines, we examined the ability of a range of cytokines to block apoptosis of VSMCs in low serum conditions. Individual cytokines were added back to plaque and normal VSMCs over a $10^{3}$-fold concentration range. Cell death was monitored and quantified by time-lapse videomicroscopy. The addition of IGF1, PDGF-AA, or PDGF-BB markedly suppressed apoptosis of both plaque-derived and normal VSMCs in the absence of any other exogenous cytokines or nutrients (Fig. 6, $A$ and $B$ ). In plaque-derived VSMCs, the antiapoptotic effects of IGF-1 and PDGF were titratable and observed at levels similar to those at which each cytokine is mitogenic (10-100 and 1-10 ng/ml, respectively) (Fig. $6 \mathrm{~A}$ ). In normal coronary and aortic VSMCs, IGF-1 and PDGF also suppressed cell death, but due to the 

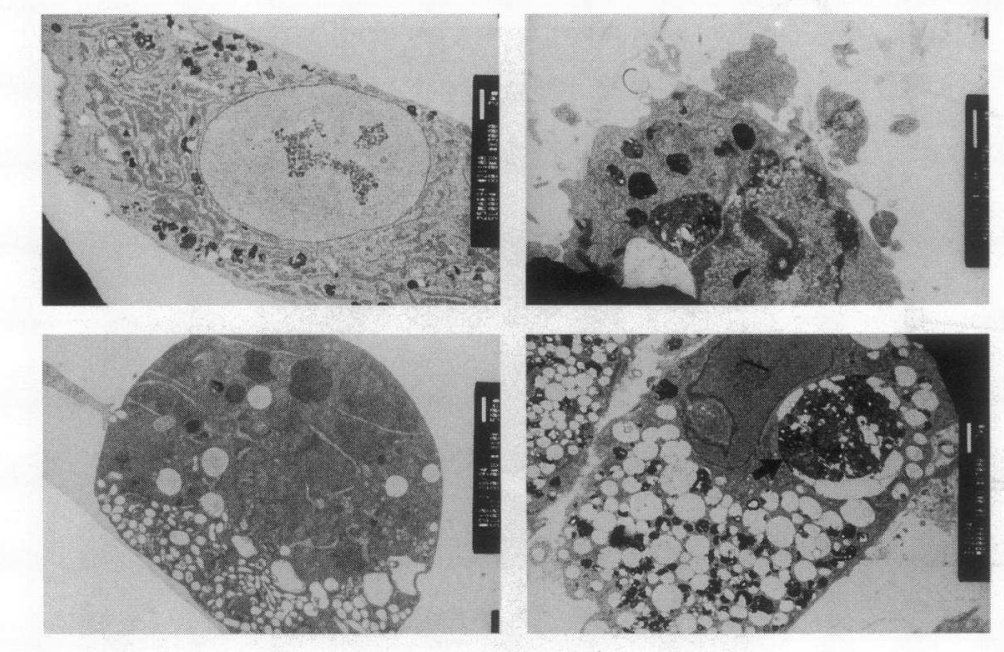

D
Figure 4. Electron microscopic appearance of apoptosis in plaque-derived human VSMCs. $(A)$ Plaque VSMC exhibiting normal morphology $(\times 3,000)$. (B) Cellular shrinkage, membrane blebbing, and formation of vesicles is evident with chromatin condensation at the periphery of the nucleus $(\times 7,500)$. (C) An apoptotic body $(\times 10,000)$. (D) An apoptotic body inside a cell of normal morphology $(\times 3,000)$ (arrow). lower rate of apoptosis in these cells, this was significant only at the $10-100-\mathrm{ng} / \mathrm{ml}$ dose range for IGF-1 and $100 \mathrm{ng} / \mathrm{ml}$ for PDGF-BB. The data for normal coronary VSMCs are shown in Fig. $6 \mathrm{~B}$, although there was a comparable effect of each cytokine on normal aortic VSMCs (not shown). In contrast to IGF-1 and PDGF, no protective effect of EGF or bFGF was observed at any concentration of growth factor for plaque-derived or normal coronary or aortic VSMCs. As PDGF-BB,

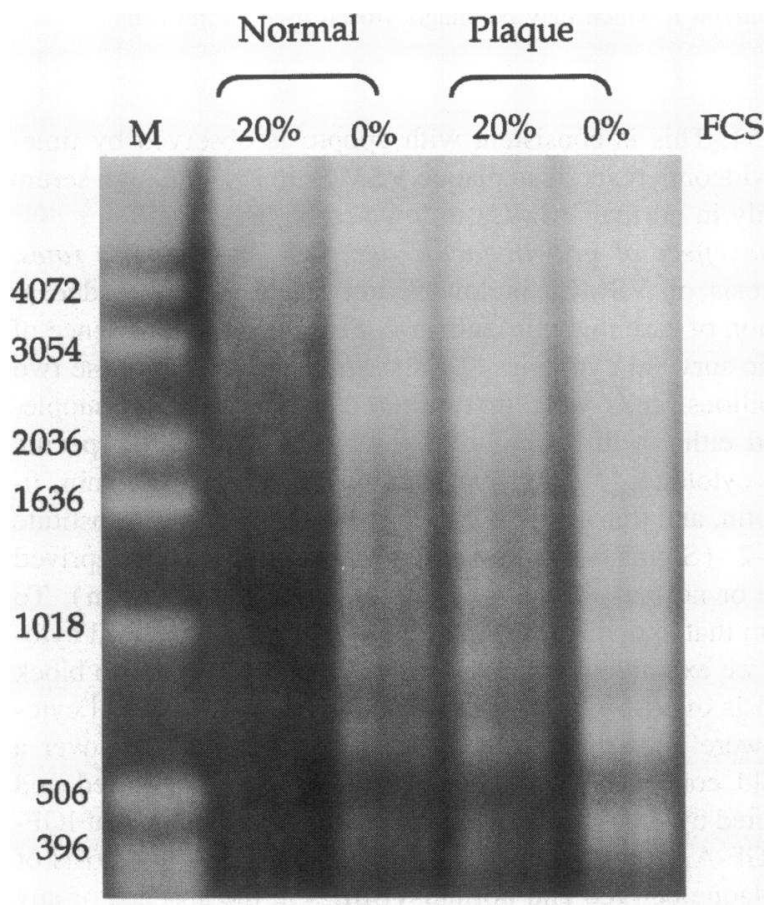

Figure 5. Electrophoresis of DNA isolated from dying cells in the supernatant of individual cultures. Normal aortic VSMCs (lane 2) and plaque-derived VSMCs (lane 4) in 20\% FCS and normal aortic VSMCs (lane 3 ) and plaque VSMCs (lane 5) in 0\% FCS are shown. DNA laddering consistent with apoptosis is evident in lanes 3-5. $M$ represents marker DNA, with size in base pairs as shown.
bFGF, and EGF are relatively potent mitogens for VSMCs in vitro, whereas IGF-1 and PDGF-AA are not (20-23), there was no obvious relationship between the potency of mitogenic action of a growth factor and its ability to suppress cell death.

The effect of bcl-2 expression on VSMC apoptosis. To investigate the effect of overexpression of $b c l-2$ on VSMC apoptosis, normal and plaque-derived VSMCs were infected with a retrovirus vector encoding human $b c l-2$ or the vector alone. Resistant cell populations were pooled after $>4$ wk selection in medium containing G418. In addition, clones of bcl-2-infected cells were obtained after 6-8 wk in culture. The rate of apoptotic death in cells containing $b c l-2$ or the vector alone was assessed by time-lapse videomicroscopy. Infection with $b c l-2$ suppressed apoptosis of normal VSMCs in low serum conditions and partially suppressed apoptosis of plaque-derived cells in both low and high serum conditions (Fig. $7 A$ ). Clones of $b c l-2$-infected cells and the pooled populations of resistant cells behaved similarly (not shown). Cells containing the vector alone had similar rates of apoptosis as uninfected cells (Fig. $7 \mathrm{~B}$ ), indicating that the protective effect of $b c l-2$ was not due to selection of cells with a decreased capacity to die in culture. $B c l-2$ infection did not immortalize the cultures of VSMCs, although it did prolong the lifespan of plaque cultures by approximately two to three passages. This effect was due to suppression of cell death alone, as bcl-2 infection had no effect on the intermitotic time of plaque cells (Table II) or the percentage of cells within the population that underwent mitosis (not shown).

The expression of bcl-2 in normal and plaque-derived VSMCs. Constitutive expression of $b c l-2$ in human plaque-derived VSMCs reduced rates of cell death to almost those seen in cells derived from normal vessels. To examine whether expression of $b c l-2$ per se could account for the differences in apoptotic rates observed between normal and plaque cells, we assessed $b c l-2$ expression in normal aortic VSMCs and plaquederived cells by Northern hybridization and immunocytochemistry. $B c l-2$ mRNA was not detectable on Northern hybridization from either plaque-derived or normal aortic VSMCs (Fig. 8). In addition, Bcl-2 protein was not seen on immunocytochemistry in VSMCs derived from plaques or normal aorta (Fig. 9) or normal coronary arteries (not shown), except in cells infected 


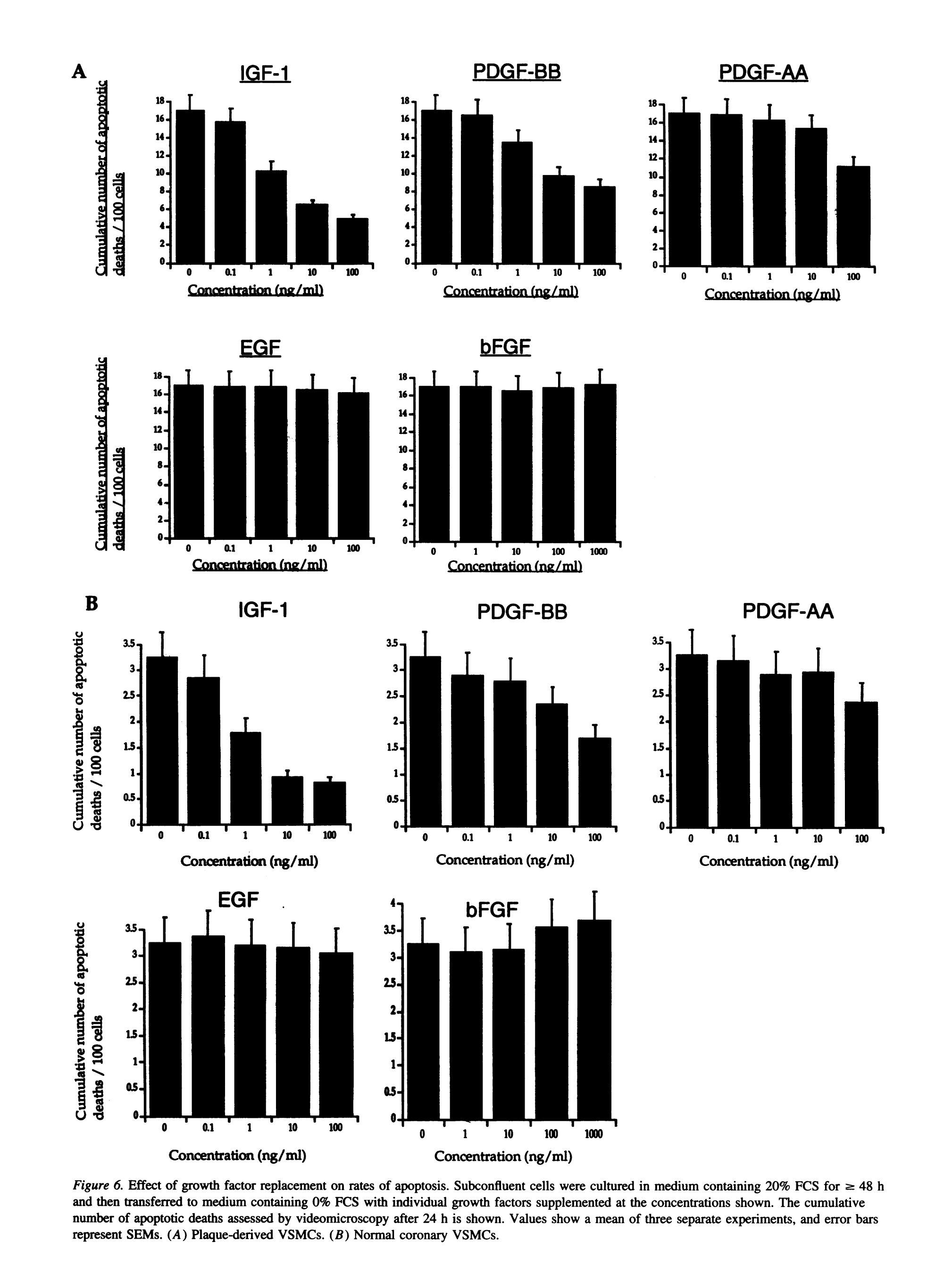


$\mathbf{A}$

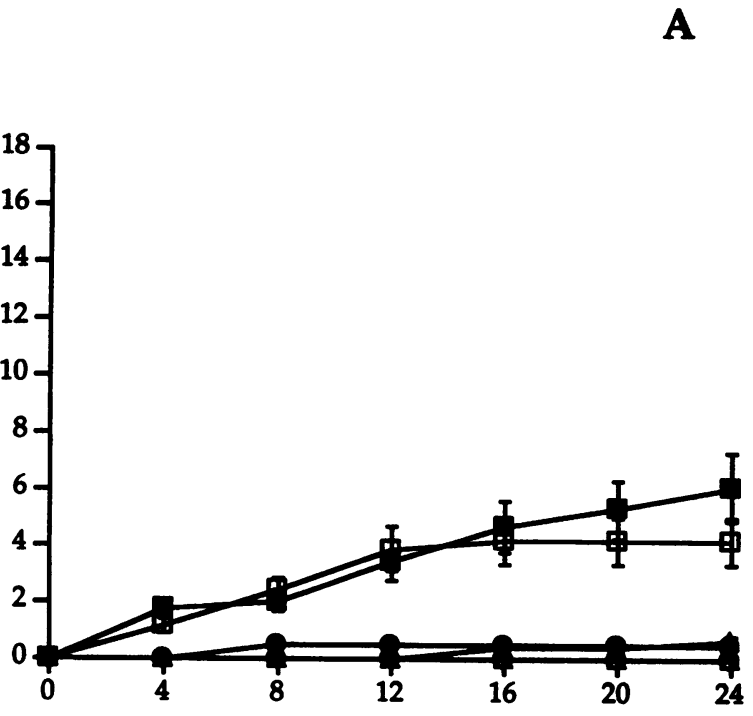

Time in hours

\section{B}

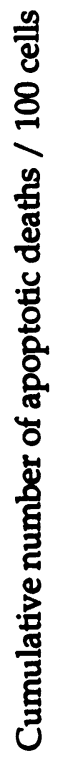

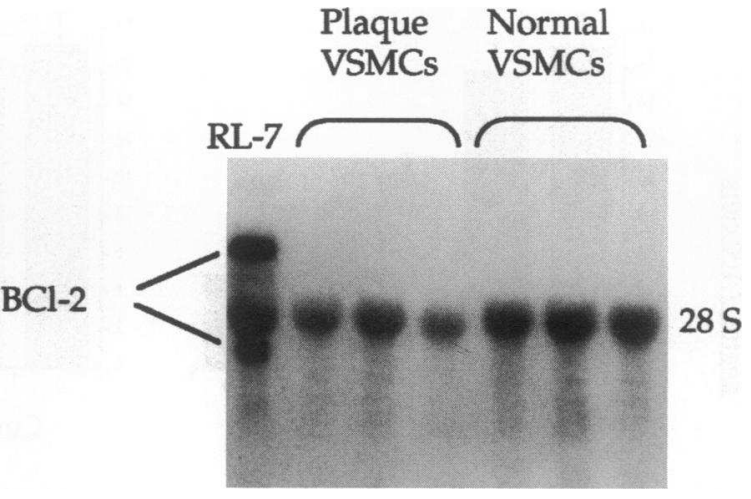

Figure 8. Northern hybridization of plaque and normal VSMCs for $b c l$ 2. Cells were cultured in 20\% FCS and RNA isolated at 50-70\% confluence. $B c l-2$ transcripts are seen in RL-7 cells (a human B-cell lymphoma cell line) at $\sim 6.5$ and $3.5 \mathrm{~kb}$. No evidence of $b c l-2$ expression was seen in three separate cultures of plaque or normal aortic VSMCs. Blots were also incubated in the presence of a probe recognising the $28 \mathrm{~S}$ ribosomal RNA band for confirmation of equal loading.

with the $b c l-2$ retrovirus vector. In infected cells, Bcl-2 was evident as a characteristic punctate cytoplasmic staining in the cytoplasm and around the periphery of the nucleus as previously described (Fig. 9) (15, 24). As Bcl-2 protected against apoptosis at levels that were easily detectable by immunocytochemistry and $b c l-2$ suppressed apoptosis in both plaque-derived and normal VSMCs, the differences we observe between rates of apoptosis in plaque and normal VSMCs are unlikely to be due to differences in endogenous Bcl-2 expression.

\section{Discussion}

We report a marked difference in spontaneous cell death in cultures of smooth muscle cells derived from human coronary atheromatous plaques as compared with cells derived from the normal vessel wall. This cell death has the characteristic mor-

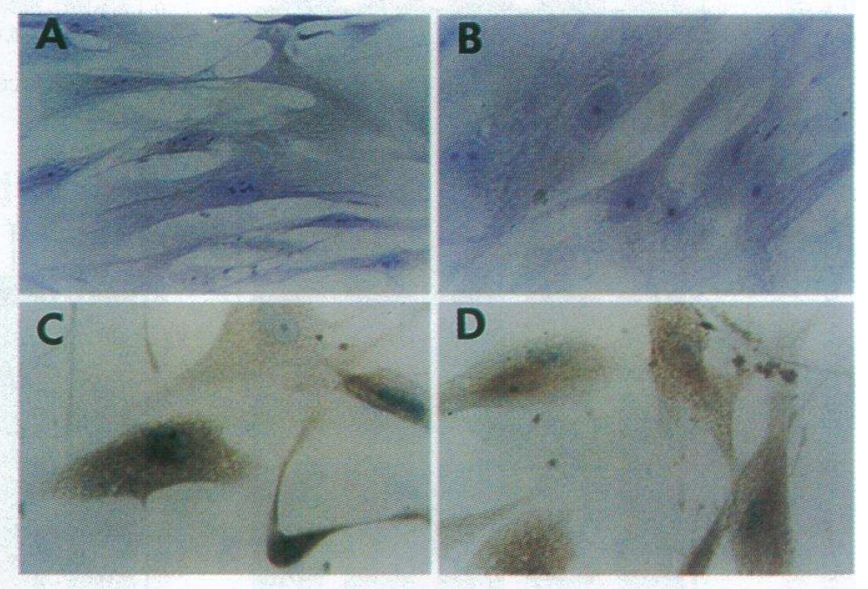

Figure 9. Immunocytochemistry for Bcl-2 protein in normal or plaque VSMCs. No evidence of Bcl-2 protein expression is seen in uninfected $(A)$ normal aortic and $(B)$ plaque-derived VSMCs. In contrast, Bcl-2 is seen as a punctate cytoplasmic staining with some perinuclear staining in cells infected with the $b c l-2$ retrovirus, $(C)$ normal aortic VSMCs, and $(D)$ plaque VSMCs. 
phological and biochemical features of apoptosis. Apoptosis occurred in cells derived from normal aortic and coronary media as well as cells from plaques, indicating that the program initiating cell death is a normal component of the cellular machinery. Apoptosis of VSMCs could be attenuated by expression of $b c l$ 2 or by provision of specific cytokines acting as survival factors.

We first investigated baseline cell kinetics of cells derived from plaques, normal aorta, and normal coronary arteries. At passages four through six, cells from plaques showed a lower rate of increase of cell number, a lower percentage of cells in S-phase, and a longer intermitotic time for individual cells than cells from either normal artery. Thus, plaque cells derived from primary coronary lesions proliferate slower in culture than normal medial smooth muscle cells at the same passage, as well as having a higher rate of cell death (see below). Both of these characteristics limit the rate of increase of cell number in culture. Although it is theoretically possible that cell death in one phase of the cell cycle accounts for the observed increased rate of cell death in the plaque-derived cells, we have previously demonstrated that apoptosis in mesenchymal cells such as fibroblasts is not associated with any one cell cycle phase (13). We therefore consider this possibility unlikely.

Cell death in plaques has previously been presumed to be due to direct toxicity of local factors, for example, oxidized lipids. However, as culture conditions of cells derived from plaques or normal vessels were identical and cells were separated from other components of the plaque, the higher death rate of plaque cells we observe implies that these VSMCs possess an innate susceptibility to cell death. As plaques show evidence of monoclonality (25), suggesting that they have arisen from a subpopulation of cells within the arterial wall, this susceptibility may be intrinsic to a cell lineage from which the plaque developed, may be acquired during atherogenesis, or both. Apoptosis in mammalian cells has been shown to be regulated by the expression of specific gene products, many of which are transcription factors (for review see reference 26). Indeed, we have previously shown that apoptosis in VSMCs may be regulated by expression of the c-myc protooncogene $(10,13)$. Here we show that human VSMC apoptosis is suppressed by expression of the protooncogene $b c l-2 . B c l-2$ is a membrane-associated protein that suppresses apoptosis in some cell types and after some injurious agents (27), including c-myc-induced apoptosis in VSMCs (our unpublished observations) and fibroblasts (28). The mechanism of $b c l-2$ action is unknown, but $b c l-2$ expression can inhibit apoptosis due to free radicals and lipid peroxidation (29), both of which are implicated in atherogenesis (8). Thus, it is conceivable that higher rates of apoptosis in plaque VSMCs is the result of some heritable presumably genetic change produced by exposure to oxidized lipids and other free radicals.

The effects of $b c l-2$ we observe are not due to clonal selection of cells with reduced susceptibility to death as death rates were identical in clones and parent populations of bcl-2-infected cells. In addition, cells infected with the retrovirus vector alone showed comparable rates of apoptosis to uninfected cells. Selection was also not due to the presence of faster replicating cells in $b c l-2$-infected populations as $b c l-2$ did not affect cell proliferation of either cell type, as assessed by rate of increase of cell number, S-phase percentage, or intermitotic time. However, it is interesting to note that whereas $b c l-2$ did not immortalize the cultures of either plaque or normal VSMCs, it did increase the ability to expand plaque cultures. Human plaquederived VSMCs in culture have previously been shown to have a finite lifespan, demonstrating a failure of culture expansion at two to five passages (30-32). This has been taken as evidence that plaque VSMCs may have undergone more doublings in vivo than cells from normal arteries. Our observations indicate that failure of plaque cell culture is at least partly due to cell death. The reported shortened lifespan of plaque VSMCs in culture may therefore be due to the greater number of cell doublings required to produce passageable cell numbers in the presence of a higher apoptotic rate.

The proportion of cells that underwent apoptosis did not change significantly in plaque-derived cells cultured in $20 \%$ FCS over passages four through six. This is despite a fairly constant rate of cell death of $7-8 \% / d$. We have previously observed that in a clonal cell line of smooth muscle cells (VSMmyc cells, rat smooth muscle cells possessing deregulated expression of the c-myc protooncogene [10]) that is forced to undergo repeated rounds of apoptosis, the remaining cells undergo apoptosis at the same rate throughout (our unpublished observations). This argues that apoptosis in our VSMC cultures may be a stochastic process, an ability possessed by all cells in the culture and not limited to a particular subset of smooth muscle cells. This does not, of course, preclude the possibility that a subset of VSMCs is present in the vessel wall possessing an increased susceptibility to apoptosis.

We also investigated the role of specific growth factors present in serum to regulate apoptosis of human VSMCs. We show that normal human VSMCs undergo apoptosis upon serum removal, and death can be suppressed by addition of IGF-1 and to a lesser extent PDGF. This is consistent with recent findings indicating that IGF-1 and PDGF are potent survival factors for both rat fibroblasts and VSMCs when death is induced by the $c-m y c$ protooncogene $(10,13)$. The requirement for survival factors indicates that cells of the normal vessel wall require constant exposure to such factors to maintain viability. Although we have demonstrated this requirement in vitro, the presence of growth factors in the vessel wall in vivo may also be necessary for cell survival.

In contrast to normal VSMCs, plaque VSMCs show spontaneous cell death, even in the presence of high concentrations of serum survival factors, and death is further promoted by serum removal. Thus, apoptosis may occur in vivo in plaques even when cells are constantly exposed to high concentrations of survival cytokines. Given the absence of elevated rates of VSMC replication in plaques $(33,34)$, even in those plaques that contain increased amounts of growth factors, our data suggest that growth factors present in lesions might be important to stabilize lesions rather than promoting growth of the plaque. Thus, cell loss via apoptosis may ultimately predispose to plaque fissuring or rupture, events closely associated with major clinical sequelae such as thrombosis and myocardial infarction (35).

Although higher rates of apoptosis-may be a pathological property of plaque VSMCs, apoptosis is also a homeostatic mechanism of maintaining cell number, particularly important in development and tissue remodeling. Our findings suggest that apoptosis may be a principal regulator of cell number in the vessel wall, balancing cell proliferation and thus helping to maintain vessel wall architecture. In support of this hypothesis is recent evidence indicating that apoptosis is a major determinant of VSMC cell number in the arterial wall after birth, where major remodeling occurs due to changes in blood flow (36). In addition, cell kinetic studies indicate a tight link between 
VSMC birth and death rates seen in normal arteries (4), a link that may be lost upon development of atherosclerosis. Changes in the rates of cell death may therefore be a mechanism by which cells accumulate or are lost, irrespective of any changes in proliferation.

In conclusion, we have demonstrated that human VSMCs derived from normal vessels and atheromatous plaques undergo apoptosis, particularly upon removal of serum survival factors. Apoptosis may be suppressed by expression of $b c l-2$ or the addition of specific survival cytokines, indicating that apoptosis of VSMCs is regulated by the expression of specific gene products and by the local cytokine environment. Apoptosis may therefore be a mechanism of regulating cell number in the normal arterial wall and contribute to the pathogenesis of atherosclerosis.

\section{Acknowledgments}

We thank Dr. David Hockenbery and Dr. A. D. Miller, Fred Hutchison Cancer Center, Seattle, for the $b c l-2$ and LXSN retrovirus vectors, respectively, and Dr. Hockenbery for the SFFV-bcl-2 cDNA, anti-Bcl-2 antibody, and the RL-7 cells. We also thank Stephanie Lara and Rene Collman for the electron microscopy.

M.R.B. is supported by a British Heart Foundation Clinical Scientist Fellowship. This study was also supported by National Institutes of Health grant HI-47151.

\section{References}

1. Garratt, K. N., W. D. Edwards, U. P. Kaufmann, R. E. Vlietstra, and D. R. J. Holmes. 1991. Differential histopathology of primary atherosclerotic and restenotic lesions in coronary arteries and saphenous vein bypass grafts: analysis of tissue obtained from 73 patients by directional atherectomy. J. Am. Coll. Cardiol. 17:442-448.

2. Arbustini, E., M. Grasso, M. Diegoli, A. Pucci, M. Bramerio, D. Ardissino, L. Angoli, S. de Seriv, E. Bramucci, A. Mussini, et al. 1991. Coronary atherosclerotic plaques with and without thrombus in ischemic heart syndromes: a morphologic, immunohistochemical, and biochemical study. Am. J. Cardiol. 68:36B$50 \mathrm{~B}$.

3. Davies, M. J. 1990. A macro and micro view of coronary vascular insult in ischemic heart disease. Circulation. 82(Suppl. 2):II-38-II-46.

4. Thomas, W. A., J. M. Reiner, F. A. Florentin, K. T. Lee, and W. M. Lee. 1976. Population dynamics of arterial smooth muscle cells. V. Cell proliferation and cell death during initial 3 months in atherosclerotic lesions induced in swine by hypercholesterolemic diet and intimal trauma. Exp. Mol. Pathol. 24:360-374.

5. Thomas, W. A., R. F. Scott, R. A. Florentin, J. M. Reiner, and K. T. Lee. 1981. Population dynamics of arterial cells during atherogenesis. XI. Slowdown in multiplication and death rates of lesion smooth muscle cells in swine during the period 105-165 days after balloon endothelial cell denudation followed by a hyperlipidemic diet. Exp. Mol. Pathol. 35:153-162.

6. Thomas, W., D. Kim, K. Lee, J. Reiner, and J. Schmee. 1983. Population dynamics of arterial cells during atherogenesis. XIII. Mitogenic and cytotoxic effects of a hyperlipidaemic (HL) diet on cells in advanced lesions in the abdominal aortas of swine fed an HL diet for 270-345 days. Exp. Mol. Pathol. 39:257270.

7. Guyton, J. R., B. L. Black, and C. L. Seidel. 1990. Focal toxicity of oxysterols in vascular smooth muscle cell culture. A model of the atherosclerotic core region. Am. J. Pathol. 137:425-434.

8. Esterbauer, H., G. Wag, and H. Puhl. 1993. Lipid peroxidation and its role in atherosclerosis. Br. Med. Bull. 49:566-576.

9. Araki, S., Y. Shimada, K. Kaji, and H. Hayashi. 1990. Apoptosis of vascular endothelial cells by fibroblast growth factor deprivation. Biochem. Biophys. Res. Commun. 168:1194-1200.

10. Bennett, M. R., G. I. Evan, and A. C. Newby. 1994. Deregulated c-myc oncogene expression blocks vascular smooth muscle cell inhibition mediated by heparin, interferon- $\gamma$, mitogen depletion and cyclic nucleotide analogues and induces apoptotic cell death. Circ. Res. 74:525-536.

11. Reid, V. C., S. J. Hardwick, and M. J. Mitchinson. 1993. Fragmentation of DNA in P388D1 macrophages exposed to oxidised low-density lipoprotein. FEBS (Fed. Eur. Biochem. Soc.) Lett. 332:218-220.

12. Wyllie, A. H. 1992. Apoptosis and the regulation of cell numbers in normal and neoplastic tissues: an overview. Cancer Metastosis Rev. 11:95-103.

13. Harrington, E., M. Bennett, A. Fanidi, and G. Evan. 1994. c-Myc induced apoptosis in fibroblasts is inhibited by specific cytokines. EMBO (Eur. Mol. Biol. Organ.) J. 13:3286-3295.

14. Parkes, J. L., R. R. Cardell, F. C. Hubbard, D. Hubbard, A. Meltzer, and A. Penn. 1991. Cultured human atherosclerotic plaque smooth muscle cells retain transforming potential and display enhanced expression of the myc protooncogene. Am. J. Pathol. 138:765-775.

15. Hockenbery, D., G. Nunez, C. Milliman, R. D. Schreiber, and S. J. Korsmeyer. 1990. Bcl-2 is an inner mitochondrial membrane protein that blocks programmed cell death. Nature. 348:334-336.

16. Evan, G. I., A. H. Wyllie, C. S. Gilbert, T. D. Littlewood, H. Land, M. Brooks, C. M. Waters, L. Z. Penn, and D. C. Hancock. 1992. Induction of apoptosis in fibroblasts by c-myc protein. Cell. 69:119-128.

17. Chirgwin, J. M., A. E. Przybyla, R. J. MacDonald, and W. J. Rutter. 1979. Isolation of biologically active ribonucleic acid from sources enriched in ribonuclease. Biochemistry. 18:5294-5299.

18. Bennett, M. R., S. Anglin, J. R. McEwan, R. Jagoe, A. C. Newby, and G. I. Evan. 1994. Inhibition of vascular smooth muscle cell proliferation in vitro and in vivo by c-myc antisense oligodeoxynucleotides. J. Clin. Invest. 93:820828.

19. Wyllie, A. H. 1987. Apoptosis: cell death in tissue regulation. J. Pathol. 153:313-316.

20. Hwang, D. L., L. J. Latus, and R. A. Lev. 1992. Effects of plateletcontained growth factors (PDGF, EGF, IGF-I, and TGF-beta) on DNA synthesis in porcine aortic smooth muscle cells in culture. Exp. Cell Res. 200:358-360.

21. Schollmann, C., R. Grugel, D. Tatje, J. Hoppe, J. Folkman, D. Marme, and H. Weich. 1992. Basic fibroblast growth factor modulates the mitogenic potency of the platelet-derived growth factor (PDGF) isoforms by specific upregulation of the PDGF alpha receptor in vascular smooth muscle cells. J. Biol. Chem. 267:18032-18039.

22. Inui, H., Y. Kitami, T. Kondo, and T. Inagami. 1993. Transduction of mitogenic activity of platelet-derived growth factor (PDGF) AB by PDGF-beta receptor without participation of PDGF-alpha receptor in vascular smooth muscle cells. J. Biol. Chem. 268:17045-17050.

23. Grotendorst, G. R., A. Igarashi, R. Larson, Y. Soma, and M. Charette. 1991. Differential binding, biological and biochemical actions of recombinant PDGF AA, AB, and BB molecules on connective tissue cells. J. Cell. Physiol. 149:235-243.

24. Jacobson, M. D., J. F. Burne, M. P. King, T. Miyashita, J. C. Reed, and M. C. Raff. 1993. Bcl-2 blocks apoptosis in cells lacking mitochondrial DNA. Nature (Lond.). 361:365-369.

25. Benditt, E. P., and J. M. Benditt. 1973. Evidence for a monoclonal origin of human atherosclerotic plaques. Proc. Natl. Acad. Sci. USA. 70:1753-1756.

26. Bennett, M., and G. Evan. 1994. The molecular basis of apoptosis. Heart Failure. 9:199-212.

27. Reed, J. 1994. Bcl-2 and the regulation of programmed cell death. J. Cell Biol. 124:1-6.

28. Fanidi, A., E. Harrington, and G. Evan. 1992. Cooperative interaction between c-myc and bcl-2 proto-oncogenes. Nature (Lond.). 359:554-556.

29. Hockenbery, D. M., Z. N. Oltvai, X. M. Yin, C. L. Milliman, and Korsmeyer S. J. 1993. Bcl-2 functions in an antioxidant pathway to prevent apoptosis. Cell. 75:241-251.

30. Moss, N., and E. Benditt. 1973. Human atherosclerotic plaque cells and leiomyoma cells. Comparison of in vitro growth characteristics. Am. J. Pathol. 78:175-90.

31. Ross, R., T. N. Wight, E. Strandness, and B. Thiele. 1984. Human athero sclerosis. I. Cell constitution and characteristics of advanced lesions of the superficial femoral artery. Am. J. Pathol. 114:79-93.

32. Dartsch, P. C., R. Voisard, and E. Betz. 1990. In vitro growth characteristics of human atherosclerotic plaque cells: comparison of cells from primary stenosing and restenosing lesions of peripheral and coronary arteries. Res. Exp. Med. Berl. 190:77-87.

33. Gordon, D., M. A. Reidy, E. P. Benditt, and S. M. Schwartz. 1990. Cell proliferation in human coronary arteries. Proc. Natl. Acad. Sci. USA. 87:46004604.

34. O'Brien, E. R., C. E. Alpers, D. K. Stewart, M. Ferguson, N. Tran, D. Gordon, E. P. Benditt, T. Hinohara, J. B. Simpson, and S. M. Schwartz. 1993. Proliferation in primary and restenotic coronary atherectomy tissue. Implications for antiproliferative therapy. Circ. Res. 73:223-231.

35. Davies, M. J. 1992. Anatomic features in victims of sudden coronary death. Coronary artery pathology. Circulation. 85(1 Suppl.):119-124.

36. Cho, A., D. Courtman, and L. Langille. 1995. Apoptosis (programmed cell death) in arteries of the neonatal lamb. Circ. Res. 76:168-175. 